\title{
EFEITO DO TREINAMENTO FÍSICO AERÓBICO EM CORONARIOPATAS SUBMETIDOS A UM PROGRAMA DE REABILITAÇÃO CARDIOVASCULAR
}

\author{
EFFECT OF AEROBIC PHYSICAL TRAINING IN PACIENTS WITH CORONARY ARTERY \\ DISEASE PARTICIPANTS OF A CARDIAC REHABILITATION PROGRAM
}

Maurício Milani ${ }^{1}$, Renata T Kozuki ${ }^{2}$, Júlio C Crescêncio ${ }^{3}$, Valéria Papa ${ }^{2}$, Michele DB Santos ${ }^{2}$, Camila Q Bertini², Cristiana AF Amato ${ }^{4}$, Vanessa CR Miranda², Fabio G Flosi ${ }^{1}$, Nataly L Izeli ${ }^{2}$, Benedito C Maciel ${ }^{5}$, Lourenço Gallo Junior ${ }^{5}$

\footnotetext{
${ }^{1}$ Médico. ${ }^{2}$ Fisioterapeuta. ${ }^{3}$ Biólogo. ${ }^{4}$ Nutricionista. ${ }^{5}$ Docente. Divisão de Cardiologia. Departamento de Clínica Médica. Faculdade de Medicina de Ribeirão Preto - USP

CoRrespondência: Lourenço Gallo Jr - Igjunior@fmrp.usp.br. Divisão de Cardiologia. Departamento de Clínica Médica. Faculdade de Medicina de Ribeirão Preto - USP. Campus Universitário-USP. 14048-900 - Ribeirão Preto - SP.
}

Milani $\mathrm{M}$ et al. Efeito do treinamento físico aeróbico em coronariopatas submetidos a um Programa de Reabilitação Cardiovascular. Medicina (Ribeirão Preto) 2007;40 (3): 403-11, jul./set.

Resumo: Modelo do estudo: Retrospectivo. Objetivos do estudo: Avaliar o efeito do treinamento físico aeróbico nas variáveis cardiovasculares em pacientes coronariopatas participantes do Programa de Reabilitação Cardiovascular. Metodologia: Foram incluídos pacientes portadores de coronariopatia estável e foram avaliadas as variáveis cardiovasculares de testes ergométricos seriados, realizados antes e após um período mínimo de 12 semanas de treinamento físico aeróbico em um Programa de Reabilitação Cardiovascular, de fevereiro de 2002 a julho de 2005. Resultados: Documentou-se incremento significativo $(p<0,0001)$ do consumo de oxigênio pico $\left(\mathrm{VO}_{2}\right.$ pico) após a reabilitação cardiovascular (30,1 $\pm 9,5$ versus $\left.35,5 \pm 8,8 \mathrm{ml} / \mathrm{kg} / \mathrm{min}\right)$. Este delta do $\mathrm{VO}_{2}$ pico apresentou correlação negativa com a capacidade física inicial, com maiores ganhos nos pacientes com menores valores iniciais de $\mathrm{VO}_{2}$ pico. Não foram observadas diferenças significativas na pressão arterial sistólica e no duplo produto pico, e uma diferença de pequena magnitude foi observada na freqüência cardíaca pico. A melhora no limiar isquêmico do miocárdio, avaliada pelo consumo de oxigênio na positivação, foi ainda mais expressiva, com incremento de $7,4 \mathrm{ml} / \mathrm{kg} /$ $\min (p<0,0001)(21,0 \pm 6,9$ versus $28,4 \pm 8,2 \mathrm{ml} / \mathrm{kg} / \mathrm{min})$. Conclusões: A reabilitação cardiovascular melhorou a capacidade física e o limiar isquêmico de pacientes participantes do Programa de Reabilitação Cardiovascular. O benefício na capacidade física foi maior nos pacientes com menores valores iniciais de consumo de oxigênio pico.

Descritores Doenças Cardiovasculares. Reabilitação. Coronariopatia. Exercício Aeróbico.

\section{1- INTRODUÇÃO}

A coronariopatia ou doença arterial coronariana (DAC) é a maior causa de morbidade e mortalidade na maioria dos países industrializados. A doença é decorrente da aterosclerose, um processo degenerativo vascular, que acomete as artérias coronarianas, levando a obstruções da luz arterial, o que pode comprometer o fluxo sangüíneo em esforço ou repouso. O desenvolvimento da doença está associado ao estilo de vida 
e à presença de fatores de risco cardiovasculares, como gênero masculino, idade maior que 45 anos, sedentarismo, presença de hipertensão arterial, diabete melito, dislipidemia, tabagismo, obesidade e história familiar de DAC precoce..$^{1,2}$

A angina estável é uma síndrome caracterizada por sensação de dor e/ou desconforto precordial ou torácico, tipicamente desencadeada ou agravada por esforço físico ou estresse emocional, e aliviada por repouso ou uso de nitrato sublingual. Esta sintomatologia ocorre pela isquemia miocárdica, que é o desequilíbrio entre a oferta e o consumo de oxigênio no miocárdio devido, geralmente, à presença de uma obstrução coronariana epicárdica (acima de 70\% do diâmetro do vaso), que pode impedir o aumento de fluxo coronariano em situação de esforço físico ou estresse emocional. O comprometimento do fluxo coronariano em repouso pode levar a quadros de angina instável e infarto agudo do miocárdio. ${ }^{1,2}$

$\mathrm{O}$ tratamento da DAC na fase crônica envolve o controle clínico da doença e dos fatores de risco cardiovasculares por meio de abordagem farmacológica, revascularização coronariana, quando necessária, e modificações do estilo de vida. Estas devem ser obtidas por meio da adoção de hábitos saudáveis, dieta equilibrada, interrupção do tabagismo, redução da massa corporal e prática regular de exercícios físi$\cos ^{1,2}$ A prática de exercícios físicos é considerada fundamental dentro do tratamento não-farmacológico, e deve ser implementada por um programa de reabilitação cardiovascular. ${ }^{3}$

A reabilitação cardiovascular é definida como um ramo de atuação da cardiologia que, implementada por uma equipe multiprofissional, permite a restituição, ao indivíduo, de uma satisfatória condição clínica, psicológica e laborativa. ${ }^{3}$ Programas de reabilitação cardiovascular consistem de uma abordagem individualizada dos pacientes com objetivos globais que visam à redução do risco cardiovascular e a melhora da qualidade de vida. Esta abordagem deve ser realizada pela equipe multiprofissional com ênfase no controle dos fatores de risco, redução de incapacidades físicas e adoção de comportamentos saudáveis, como estilo de vida ativo. Ela inclui, além do exercício físico, orientações nutricionais, abordagens psicossociais e estímulo à aderência aos procedimentos terapêuticos ${ }^{4}$

Diversos benefícios são observados com a participação regular de pacientes coronariopatas em programas de reabilitação cardiovascular, tanto no controle dos fatores de risco, ${ }^{5,6}$ quanto na melhora da sin- tomatologia, do limiar de angina, da capacidade física, da qualidade de vida e do risco cardiovascular. ${ }^{3 / 12}$ Estes benefícios observados após o treinamento físico decorrem de diversas modificações favoráveis nas artérias coronarianas, com melhora da perfusão miocárdica e, conseqüentemente, da isquemia induzida por esforço físico. ${ }^{13 / 20}$

A reabilitação cardiovascular, além dos diversos benefícios observados, apresenta elevada segurança, com baixa incidência de eventos cardiovasculares durante a prática de exercícios físicos regulares. ${ }^{21,22}$

Com os objetivos de se obter uma avaliação clínica, estratificação do risco cardiovascular e prescrição dos exercícios físicos, os pacientes coronariopatas devem realizar um teste ergométrico (TE) para se documentar a resposta das variáveis cardiovasculares ao esforço físico crescente, como a freqüência cardíaca (FC), a pressão arterial (PA) e o duplo produto (DP). ${ }^{23,24}$

Por meio da velocidade e inclinação da esteira ergométrica no pico do esforço físico, pode-se calcular o consumo de oxigênio $\left(\mathrm{VO}_{2}\right.$ pico), obtido indiretamente por fórmulas ${ }^{24}$. $\mathrm{O} \mathrm{VO}_{2}$ pico é um parâmetro que reflete a aptidão cardiorrespiratória. A captação pico de oxigênio é o produto de débito cardíaco pico (L/min) pela diferença do conteúdo arteriovenoso de oxigênio $\left(\mathrm{mL}\right.$ de $\left.\mathrm{O}_{2}\right)$, sendo que o débito cardíaco depende da FC (batimento/min) e do volume sistólico (mL/batimento) ejetado pelo ventrículo esquerdo (VS). As diferenças individuais nos valores do $\mathrm{VO}_{2}$ pico são devidas, principalmente, às diferenças no débito cardíaco máximo e, portanto, $\mathrm{o} \mathrm{VO}_{2}$ está ligado à capacidade funcional do coração, principalmente em cardiopatas. ${ }^{24}$

$\mathrm{O} \mathrm{VO}_{2}$ pico é um importante índice prognóstico, com correlação negativa com mortalidade cardiovascular e geral, isto é, menores valores de capacidade física estão correlacionados a maior mortalidade..$^{25,26,27}$ Mudanças obtidas na capacidade física e na prática regular de exercícios físicos induzem modificações prognósticas em doentes e indivíduos saudáveis. $^{28,29,30}$

O TE pode apresentar positivação, que é a presença de alterações clínicas, hemodinâmicas e/ou eletrocardiográficas sugestivas de isquemia miocárdica. As alterações clínicas geralmente incluem a presença de precordialgia e dispnéia ao esforço físico, as hemodinâmicas se relacionam à presença de sinais de disfunção ventricular no esforço, responsáveis pela con- 
gestão pulmonar ou queda pressórica no esforço crescente, e as alterações eletrocardiográficas dizem respeito ao desenvolvimento de infradesnivelamento ou supradesnivelamento do segmento ST. ${ }^{23,24}$ As alterações sugestivas de isquemia podem iniciar em determinada carga, por vezes ainda permitindo a progressão do esforço até a interrupção do teste. A determinação da $\mathrm{FC}$ e do $\mathrm{VO}_{2}$ de positivação, na respectiva carga, é importante para se identificar o limiar isquêmico. $^{5,24}$

A partir das informações clínicas e do resultado do TE, determina-se o risco, o tipo de supervisão requerida e a prescrição das sessões de reabilitação cardiovascular. ${ }^{3,5}$ Um dos métodos para a prescrição do esforço é o uso da freqüência cardíaca de treinamento, calculada pela fórmula de Karvonen, com prescrição de exercícios aeróbicos em 50 a 70\% da FC de reserva, utilizando nesta fórmula a FC pico atingida no TE ou a FC de positivação da isquemia. ${ }^{3,5,7}$

Com o intuito de se obter os benefícios esperados com o treinamento físico em pacientes coronariopatas, foi implantado, em fevereiro de 2002, o Programa de Reabilitação Cardiovascular da Divisão de Cardiologia, do Departamento de Clínica Médica do Hospital das Clínicas da Faculdade de Medicina de Ribeirão Preto - Universidade de São Paulo (HCFMRP-USP).

O objetivo do presente estudo é avaliar o efeito do treinamento físico aeróbico nas variáveis cardiovasculares em pacientes coronariopatas participantes do Programa de Reabilitação Cardiovascular, analisando testes ergométricos seriados, realizados antes e após um período mínimo de 12 semanas de treinamento.

\section{2- CASUÍSTICA}

\section{1- Amostra}

Foi realizada uma análise retrospectiva nos pacientes coronariopatas participantes do Programa de Reabilitação Cardiovascular da Divisão de Cardiologia do Departamento de Clínica Médica do HCFMRPUSP, que iniciaram o treinamento físico entre março de 2002 a julho de 2005 e que permaneceram no Programa por um período mínimo de 12 semanas, com realização de 2 TE (antes e após o treinamento) com intervalo superior a 12 semanas.

Foram incluídos pacientes portadores de coronariopatia estável, sem eventos clínicos ou procedimentos de revascularização miocárdica nos últimos 3 meses antes do início do Programa de Reabilitação Cardiovascular.

Foram obtidas informações clínicas em relação à antropometria, presença de fatores de risco, antecedentes cardiovasculares e presença de disfunção ventricular esquerda.

\section{2- Teste ergométrico}

O TE foi realizado em esteira ergométrica no sistema Ergo PC $®$ da Micromed $\AA$, com monitorização eletrocardiográfica de 3 derivações (CM5, D2M e V2M), medidas intermitentes da pressão arterial pelo método auscultatório, anotação de eventuais queixas clínicas e alterações no exame físico. Foram utilizados protocolos contínuos, com incrementos progressivos de cargas (velocidade e inclinação) a cada minuto de esforço, ajustados de acordo com a capacidade física individual esperada. O teste foi realizado em vigência das medicações de uso habitual (beta-bloqueadores, inibidores da ECA, AAS, estatinas e outros). O teste foi conduzido até a exaustão cardiorrespiratória ou presença de critérios de interrupção do esforço. ${ }^{23,24}$ Em caso de aparecimento de sintomas isquêmicos leves, o teste continuou a ser executado até a exaustão ou a intensificação destes sintomas.

No pico do esforço foram obtidos a freqüência cardíaca (FC pico), a pressão arterial sistólica (PAS pico) e o duplo produto (DP pico). O consumo de oxigênio pico ( $\mathrm{VO}_{2}$ pico) foi obtido indiretamente por uso de fórmulas ${ }^{24}$ de acordo com a velocidade e inclinação atingidas no pico do esforço.

Foram observados critérios clínicos, eletrocardiográficos e/ou hemodinâmicos sugestivos de isquemia miocárdica ${ }^{23,24} \mathrm{e}$, em caso de presença de alguma alteração, foram obtidos $\mathrm{o} \mathrm{VO}_{2}$ e a $\mathrm{FC}$ nos quais se iniciaram estas alterações isquêmicas $\left(\mathrm{VO}_{2}\right.$ posit e FC posit).

Estas variáveis cardiovasculares foram comparadas entre si antes e após a reabilitação cardiovascular.

\section{3- Reabilitação cardiovascular}

O programa de reabilitação cardiovascular foi realizado utilizando-se exercícios aeróbicos de moderada intensidade, em 50 a $70 \%$ da FC de reserva, com base nos valores de FC pico ou FC posit obtidos no TE. A duração dos exercícios físicos foi de 20 a 30 minutos, além de fases de aquecimento inicial e desaquecimento final, com cargas de menor intensidade e duração de 5 minutos. 
Os pacientes que participaram das atividades supervisionadas realizaram 2 sessões semanais em esteira ou caminhadas na pista de atletismo. Nas sessões supervisionadas, foram controladas as cargas para se obter a FC dentro da zona de treinamento prescrita. Além das sessões supervisionadas, os pacientes foram estimulados a realizarem mais 2 sessões não-supervisionadas, com ajuste da velocidade da caminhada pela contagem da FC pelo pulso radial, buscando-se atingir a zona de treinamento.

\section{4- Análise estatística}

A análise estatística foi realizada por meio do software GraphPad InStat ${ }^{\circledR}$ versão 3.05, e foram utilizados os seguintes testes paramétricos: 1.) Comparações de duas amostras: Teste-t pareado; 2.) Comparações de três amostras: ANOVA para medidas repetidas com comparações múltiplas pelo Teste de Tukey-Kramer; 3.) Correlações entre variáveis: Correlação de Pearson e 4.) Comparação dos benefícios obtidos após divisão em subgrupos de acordo com variáveis categóricas dicotômicas: teste-t não pareado. O nível de significância foi estabelecido em $5 \%(\mathrm{p}<0,05)$

\section{3- RESULTADOS}

\section{1- Caracterização dos pacientes}

Foram avaliados 63 pacientes com predominância do gênero masculino, em 79,4\% dos pacientes estudados. A média de idade e desvio padrão foi de 57,4 $\pm 9,6$ anos.

Os fatores de risco cardiovasculares apresentaram as seguintes prevalências, em ordem decrescente: hipertensão arterial $(77,8 \%)$, dislipidemia $(68,3 \%)$, história familiar positiva para $\mathrm{DAC}(47,6)$, diabete melito (33,3\%), obesidade $(31,7 \%)$ e tabagismo atual $(14,3 \%)$. A prevalência de tabagismo prévio, em atual abstinência, foi de $42,9 \%$, portanto, o histórico de tabagismo atual ou pregresso esteve presente em $57,2 \%$ dos pacientes. O sobrepeso também foi muito prevalente nestes pacientes $(52,4 \%)$. Apenas 10 pacientes $(15,9 \%)$ estavam com o índice de massa corporal (IMC) dentro dos limites da normalidade. O IMC médio e desvio padrão foram de $28,5 \pm 3,9 \mathrm{Kg} / \mathrm{m}^{2}$.

A maioria dos pacientes apresentou antecedentes de infarto do miocárdio $(69,8 \%)$ com procedimentos de revascularização miocárdica cirúrgica $(33,3 \%)$ e de angioplastia coronária (49,2\%). A prevalência de disfunção ventricular esquerda nos pacientes foi de $19,7 \%$.

\section{2- Variáveis cardiovasculares}

Os testes ergométricos iniciais (T1) e após a reabilitação cardiovascular (T2) foram realizados em um intervalo médio de 20,2 \pm 5 semanas, e foram comparadas as variáveis cardiovasculares atingidas no pico do esforço físico, antes e após o treinamento.

As FC pico atingidas em T1 e T2 foram, respectivamente, de $121,0 \pm 19,6$ e $124,4 \pm 21,3 \mathrm{bpm}$. A diferença foi estatisticamente significativa $(p=0,005)$, porém de pequena magnitude $(+3,4 \mathrm{bpm})$. Não houve diferenças estatísticas na PAS pico e no DP pico após a reabilitação. (Tabela I)

\section{3- Consumo de oxigênio pico}

Os valores do $\mathrm{VO}_{2}$ pico em $\mathrm{T} 1$ e $\mathrm{T} 2$ foram, respectivamente, de $30,1 \pm 9,5$ e $35,5 \pm 8,8 \mathrm{ml} / \mathrm{kg} / \mathrm{min}$. A diferença foi estatisticamente significativa $(\mathrm{p}<$ 0,0001 ), com incremento médio no $\mathrm{VO}_{2}$ pico de $+5,4$ $\mathrm{ml} / \mathrm{kg} / \mathrm{min}$. (Tabela I e Figura 1)

Tabela I - Variáveis cardiovasculares antes e após a reabilitação cardiovascular nos testes ergométricos

\begin{tabular}{lcc}
\hline VARIÁVEIS & Antes & Após \\
\hline FC pico(bpm) & $121 \pm 20$ & $124 \pm 21^{*}$ \\
PAS pico (mmHg) & $166 \pm 19$ & $166 \pm 22$ \\
DPpico(bpm.mmHg) & $20.180 \pm 4.357$ & $20.803 \pm 4.740$ \\
VO $\mathbf{p i c o}(\mathbf{m l} / \mathbf{k g} / \mathbf{m i n})$ & $30,1 \pm 9,5$ & $35,5 \pm 8,8 \dagger$
\end{tabular}

Valores: média \pm desvio padrão.

$* \mathrm{p}=0,005 ; \dagger \mathrm{p}<0,0001$ 


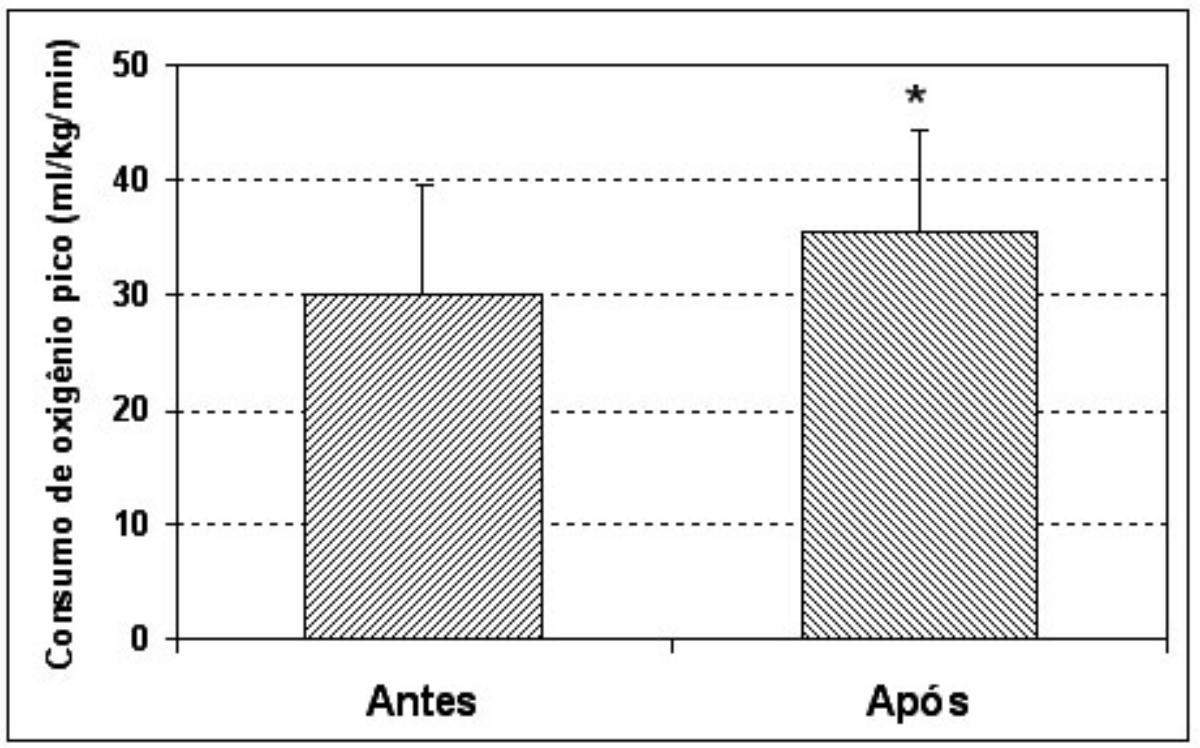

Figura 1: Consumo de oxigênio pico nos testes ergométricos antes e após a reabilitação cardiovascular. Valores: média \pm desvio padrão. $n=63$ pacientes; * $p<0,0001$.

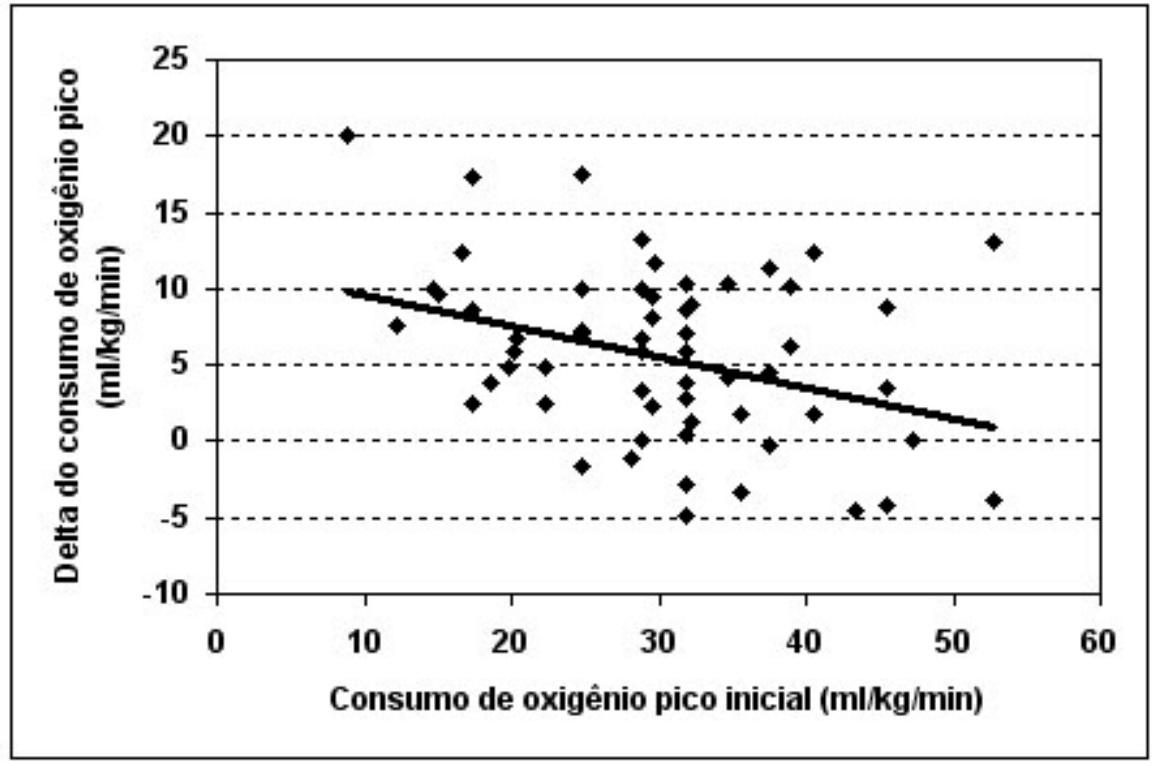

Figura 2: Correlação entre o consumo de oxigênio pico inicial e a diferença do consumo de oxigênio pico após a reabilitação cardiovascular. $n=63 ; r=-0,35 ; p=0,005$

No entanto, este benefício no $\mathrm{VO}_{2}$ pico apresentou grande heterogeneidade, com diferença oscilando entre - $4,8 \mathrm{a}+20 \mathrm{ml} / \mathrm{kg} / \mathrm{min}$. Analisando a influência das características iniciais dos pacientes sobre as diferenças do $\mathrm{VO}_{2}$ pico, observou-se correlação estatisticamente significativa apenas com o $\mathrm{VO}_{2}$ pico inicial $(r=-0,35 ; p=0,005)$. (Figura 2).
Nenhuma outra característica inicial influenciou os ganhos obtidos com a reabilitação cardiovascular. As correlações da diferença do $\mathrm{VO}_{2}$ com a idade $(\mathrm{r}=$ $0,07)$ e índice de massa corporal $(\mathrm{r}=-0,02)$, não foram estatisticamente significativas. As comparações realizadas entre as diferenças do $\mathrm{VO}_{2}$ pico, de acordo com a presença de fatores de risco, gênero, ante- 
cedentes de infarto do miocárdio e revascularizações, presença de disfunção ventricular e de isquemia miocárdica no TE inicial, também não mostraram diferenças entre os subgrupos analisados.

\section{4- Presença de isquemia miocárdica}

A presença de isquemia miocárdica foi detectada no TE inicial, por alterações clínicas, eletrocardiográficas e/ou hemodinâmicas em 26 pacientes da amostra avaliada (41,3\%). Após a reabilitação cardiovascular houve desaparecimento das alterações isquêmicas em 6 pacientes. (Tabela II)

Em relação às variáveis cardiovasculares, não foram observadas, nestes pacientes, diferenças esta- tisticamente significativas após a reabilitação na FC pico, PAS pico e DP pico. A FC posit no T1 e T2 foram, respectivamente, de 106,7 $\pm 16,9$ e 110,6 $\pm 19,0$ $\mathrm{bpm}$. A diferença não foi estatisticamente significativa. (Tabela III)

$\mathrm{O} \mathrm{VO}_{2}$ pico apresentou diferença estatisticamente significativa após a reabilitação $(\mathrm{p}<0,0001)$, com delta de $+5,9 \mathrm{ml} / \mathrm{kg} / \mathrm{min}(28,1 \pm 8,8$ versus 34,0 $\mathrm{ml} / \mathrm{kg} / \mathrm{min}$ ). Merecem destaques as modificações do $\mathrm{VO}_{2}$ no momento da ocorrência de isquemia. Assim, os valores do $\mathrm{VO}_{2}$ posit em $\mathrm{T} 1$ e $\mathrm{T} 2$ foram, respectivamente, de $21,0 \pm 6,9$ e $28,4 \pm 8,2 \mathrm{ml} / \mathrm{kg} / \mathrm{min}$, com diferença média estatisticamente significativa $(\mathrm{p}<0,0001)$, sendo de $+7,4 \mathrm{ml} / \mathrm{kg} / \mathrm{min}$. (Tabela III e Figura 3)

Tabela II: Incidência absoluta e relativa de alterações sugestivas de isquemia miocárdica nos testes ergométricos antes e após a reabilitação cardiovascular

\begin{tabular}{lcc}
\hline ISQUEMIA MIOCÁRDICA & Antes & Após \\
\hline Presente & $26(100 \%)$ & $20(76,9 \%)$ \\
Clínica isolada & $9(34,6 \%)$ & $9(34,6 \%)$ \\
Eletrocardiográfica isolada & $9(34,6 \%)$ & $8(30,8 \%)$ \\
Hemodinâmica isolada & $1(3,8 \%)$ & $1(3,8 \%)$ \\
Clínica e eletrocardiográfica & $7(26,9 \%)$ & $2(7,7 \%)$ \\
Ausente & $0(0 \%)$ & $6(23,1 \%)$ \\
\hline TOTAL & $26(100 \%)$ & $26(100 \%)$ \\
\hline
\end{tabular}

Tabela III: Variáveis cardiovasculares antes e após a reabilitação cardiovascular nos pacientes com isquemia miocárdica identificada no teste ergométrico

\begin{tabular}{lcc}
\hline VARIÁVEIS & Inicial & Após \\
\hline FC pico(bpm) & $117,7 \pm 18,8$ & $117,7 \pm 19,9$ \\
PAS pico (mmHg) & $166,5 \pm 15,6$ & $169,3 \pm 20,7$ \\
DPpico (bpm.mmHg) & $19.605 \pm 3.651$ & $20.023 \pm 4.587$ \\
VO $\mathbf{p i c o}(\mathbf{m l} / \mathbf{k g} / \mathbf{m i n})$ & $28,1 \pm 8,8$ & $34,0 \pm 7,9 *$ \\
FC posit (bpm) & $106,7 \pm 16,9$ & $110,6 \pm 19,0$ \\
VO $O_{2}$ posit (ml/kg/min) & $21,0 \pm 6,9$ & $28,4 \pm 8,2 \dagger$ \\
\hline
\end{tabular}

Valores: média \pm desvio padrão.

$* \mathrm{p}<0,0001 ; \uparrow \mathrm{p}<0,0001$ 


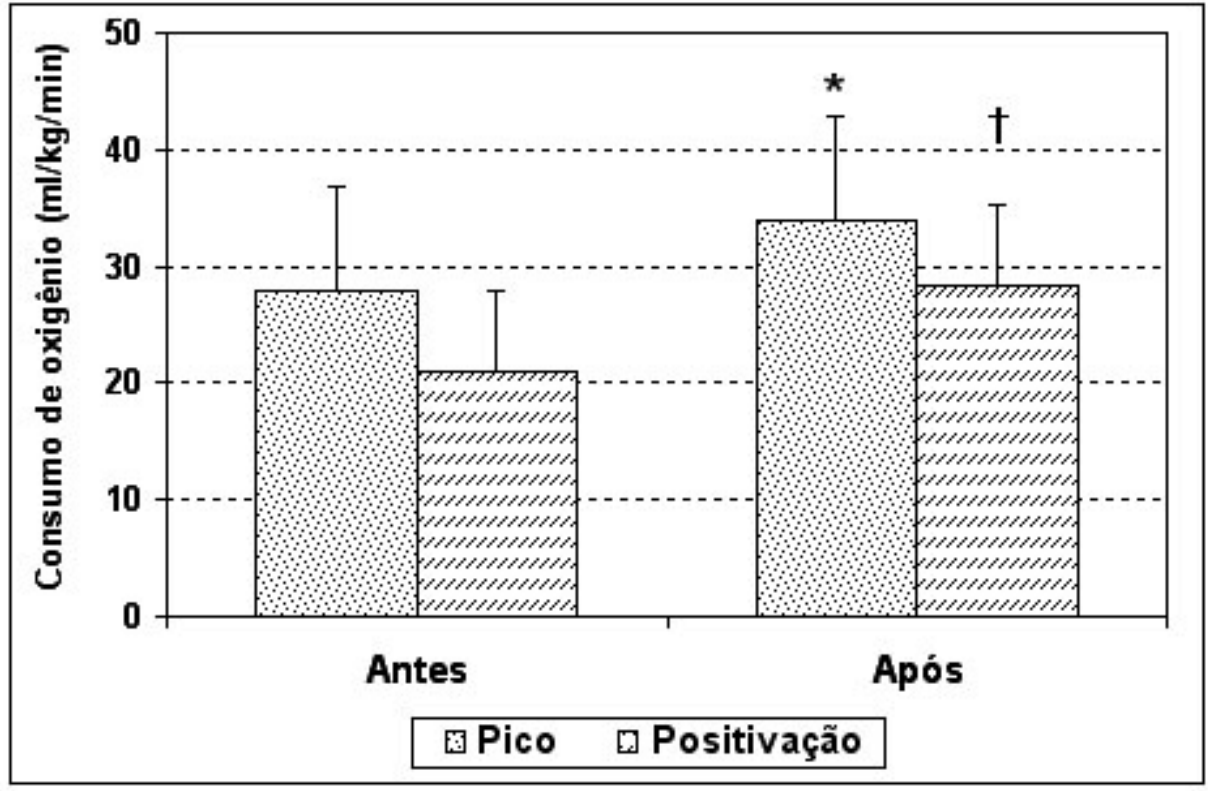

Figura 3: Consumo de oxigênio pico e positivação nos testes ergométricos antes e após a reabilitação cardiovascular nos pacientes com isquemia miocárdica.

Valores: média \pm desvio padrão. $\mathrm{n}=26$.

${ }^{*} p<0,0001$ versus valores iniciais; $\dagger p<0,0001$ versus valores iniciais.

\section{4- DISCUSSÃO}

As características da amostra de pacientes coronariopatas do presente estudo são comparáveis com a de outros estudos ${ }^{1,2}$ no que se refere à elevada prevalência de fatores de risco cardiovasculares, predomínio do gênero masculino e faixa etária acima de 50 anos.

A elevada prevalência de antecedentes de infarto do miocárdio e de revascularizações prévias demonstram uma amostra com maior gravidade da doença aterosclerótica coronariana, o que é compatível com a população de coronariopatas que freqüentam serviços de saúde terciários, como é o caso do HCFMRP-USP.

Os resultados observados no $\mathrm{VO}_{2}$ pico mostraram um dos grandes benefícios da reabilitação cardiovascular, ou seja, a melhora da capacidade física dos pacientes coronariopatas, o que está de acordo com outros estudos existentes na literatura. ${ }^{8 / 12}$ Este achado pode eventualmente propiciar uma redução do risco cardiovascular destes pacientes, visto que a capacidade física é um importante índice prognóstico. ${ }^{23,25,26}$

$\mathrm{O}$ aumento do $\mathrm{VO}_{2}$ pico observado no presente trabalho foi realmente devido a um melhor desempenho do sistema cardiovascular, visto que a sobrecarga imposta nos testes ergométricos foi equivalen- te, pois não houve diferenças significativas no DP e PAS picos e a diferença na FC pico foi de pequena magnitude, não justificando a grande melhora da capacidade física.

Os pacientes com maiores incrementos da capacidade física foram aqueles com menores valores iniciais de $\mathrm{VO}_{2}$ pico. Nenhuma outra característica influenciou este benefício do treinamento físico, o que demonstra a importância da inclusão de todos os pacientes coronariopatas estáveis em programas de reabilitação cardiovascular, principalmente daqueles pacientes mais limitados, com menores capacidades fí-

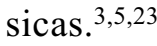

A melhora observada no limiar isquêmico dos pacientes foi um achado de grande implicação fisiopatológica. Houve negativação do teste ergométrico em 6 pacientes e o incremento do limiar isquêmico, evidenciado pelo $\mathrm{VO}_{2}$ posit, foi bastante expressivo, ou seja, de $7,4 \mathrm{ml} / \mathrm{kg} / \mathrm{min}$, o que também já foi observado em outros trabalhos, e reflete uma significativa melhora da qualidade de vida destes pacientes. 9,10,11

Esta melhora no limiar isquêmico também foi devida à melhora no desempenho do sistema cardiovascular, visto que não houve diferenças significativas no DP, PAS e FC picos, nem na FC posit, entre os dois testes realizados, antes e após o treinamento físico. 
Os mecanismos fisiopatológicos responsáveis pelo melhora observada, na capacidade física e no limiar isquêmico, não foram avaliados neste estudo, porém supõe-se que os benefícios sejam principalmente funcionais ${ }^{20}$, por melhora da função endotelial coronariana e aumento da resposta vasodilatadora ${ }^{19}$. Mecanismos adicionais relacionados ao treinamento físico ${ }^{5,6}$ devem ser considerados, como mudanças da modulação do sistema autonômico, com redução da atividade simpática em cargas submáximas, de modo a permitir uma menor FC para uma mesma carga de esforço. Alterações anatômicas, como regressões de lesões coronarianas epicárdicas e/ou formação de colaterais, são mecanismos possivelmente não respon- sáveis pela melhora observada nestes pacientes, em virtude destas alterações anatômicas requererem um tempo maior de treinamento físico contínuo para que ocorram. ${ }^{14,15,16,19}$

\section{5- CONCLUSÃO}

O treinamento físico aeróbico aplicado no presente programa de reabilitação cardiovascular foi eficiente para se obter melhora significativa na capacidade física e no limiar isquêmico dos pacientes coronariopatas. Os ganhos obtidos no $\mathrm{VO}_{2}$ pico com a reabilitação cardiovascular foram maiores quanto menor a magnitude da capacidade física inicial.

Milani $\mathrm{M}$ et al. Effect of aerobic physical training in pacients with coronary artery disease participants of a Cardiac Rehabilitation Program. Medicina (Ribeirão Preto) 2007;40 (3): 403-11, july/sept.

Abstract: Study model: Retrospective. Study objectives: Evaluate the effect of aerobic physical training in cardiovascular variables in patients with coronary artery disease participants of a Cardiovascular Rehabilitation Program. Methods: Patients with stable coronary artery disease were included and were analyzed the cardiovascular variables of exercise testing, carried out before and after a minimum period of 12 weeks of aerobic physical training in a Cardiovascular Rehabilitation Program, from February 2002 to July 2005. Results: A significant increment ( $p<$ 0.0001 ) of maximal oxygen consumption $\left(\mathrm{VO}_{2} \max \right)$ was observed after the cardiovascular rehabilitation $(30.1 \pm 9.5$ versus $35.5 \pm 8.8 \mathrm{ml} / \mathrm{kg} / \mathrm{min})$. The difference of the $\mathrm{VO}_{2}$ max had negative correlation with the initial physical capacity, with greater benefits in patients with smaller initial values of $\mathrm{VO}_{2}$ max. Significant differences were not observed in maximal systolic blood pressure and double product, and a small difference was observed in maximal heart rate. The improvement in ischemic threshold was more pronounced, with an increment of $7.4 \mathrm{ml} / \mathrm{kg} / \mathrm{min}$ in oxygen consumption, with statistical significance $(p<0.0001)(21.0 \pm 6.9$ versus $28.4 \pm 8.2 \mathrm{ml} / \mathrm{kg} / \mathrm{min})$. Conclusions: The cardiovascular rehabilitation improved physical capacity and ischemic threshold in patients participants of a Cardiovascular Rehabilitation Program. The benefit in physical capacity was greater in patients with smaller initial values of maximal oxygen consumption.

Keywords: Cardiovascular Diseases. Rehabilitation. Coronary Diseases. Exercise, Aerobic.

\section{6- REFERÊNCIAS}

1 - César LAM, editor. Diretrizes de doença coronariana crônica angina estável. Arq Bras Cardiol 2004; 83 (Suppl II): 1-43.

2 - Gibbons RJ, Abrams J, Chatterjee K, Daley J, Deedwania PC, Douglas JS, et al. ACC/AHA 2002 guideline update for the management of patients with chronic stable angina: a report of the American College of Cardiology/ American Heart Association Task Force on Practice Guidelines (Committee to Update the 1999 Guidelines for the Management of Patients with Chronic Stable Angina). 2002. Available from: http:www.acc.org/clinical/guidelines/stable/stable.pdf.
3 - Godoy M, editor. I Consenso nacional de reabilitação cardiovascular. Arq Bras Cardiol 1997; 69(4): 267-91.

4 - Balady GJ, Ades PA, Comoss P, Limacher M, Pina IL, Southard $\mathrm{D}$, et al. Core components of cardiac rehabilitation/secondary prevention programs. A Statement for healthcare professionals from the American Heart Association and the American Association of Cardiovascular and Pulmonary Rehabilitation. Circulation 2000; 102(9):1069-73.

5 - Milani M, Papa V, Gallo L Jr. Reabilitação cardiovascular. In: Maciel BC, Marin JA Neto, editores. Manual de condutas clínicas cardiológicas. São Paulo: Segmentofarma;2005. p. 287-95. 
6 - Shephard RJ, Balady GJ. Exercise as cardiovascular therapy Circulation 1999; 99(7): 963-72.

7 - Alves GB, Roveda F, Watanabe E, Nunes N, Nery SS. Reabilitação cardiovascular e condicionamento físico. In: Negrão CE, Barreto ACP, editores. Cardiologia do exercício. Barueri: Manole, 2005. p.249-59.

8 - Yu C, Sheung-Wai L, Ho HH, Lau C. Long term changes in exercise capacity, quality of life, body anthropometry, and lipid profiles after a cardiac rehabilitation program in obese patients with coronary heart disease. Am J Cardiol 2003; 91 (3): 321-5

9 - Marchionni N, Fattirolli FR, Fumagalli S, Oldridge N, Del Lungo $\mathrm{F}$, Morosi $\mathrm{L}$, et al. Improved exercise tolerance and quality of life with cardiac rehabilitation of older patients after myocardial infarction. Circulation 2003; 107(17): 2201-6.

10 - Hambrecht R, Walther C, Möbius-Winkler S, Gielen S, Linke A, Conradi K, et al. Percutaneous coronary angioplasty compared with exercise training in patients with stable coronary artery disease. A randomized trial. Circulation 2004; 109(11): 1371-8.

11 - Ehsani AA, Heath GW, Hagberg JM, Sobel BE, Holloszy JO. Effects of 12 months of intense exercise training on is chemic ST-segment depression in patients with coronary artery disease. Circulation 1981; 64(6): 1116-24.

12 - Pratt CM, Welton DE, Squires WG, Kirby TE, Hartung H, Miller RR. Demonstration of training effect during chronic â-adrenergic blockade inpatients with coronary artery disease. Circulation 1981; 64(6): 1125-9.

13 - Wannamethee SG, Shaper AG, Walker M, Physical activity and mortality in older men with diagnosed coronary heart disease. Circulation 2000; 102(12): 1358-63.

14 - Ornish D, Scherwitz LW, Billings JH, Gould KL, Merritt TA, Sparler, SMA, et al. Intensive lifestyle changes for reversal of coronary heart disease. JAMA 1998; 280(23): 2001-7.

15 - Haskell WL, Alderman EL, Fair JM, Maron DJ, Mackey SF, Superko HR, et al. Effects of intensive multiple risk factor reduction on coronary atherosclerosis and clinical cardiac events in men and women with coronary artery disease: The Stanford Coronary Risk Intervention Project (SCRIP). Circulation 1994; 89(3): 975-90.

16 - Niebauer J, Hambrecht R, Velich T, Hauer K, Marburger C, Kalberer B, et al. Attenuated progression of coronary artery disease after 6 years of multifactorial risk intervention, role of physical exercise. Circulation 1997; 96(8): 2534-41.

17 - Belardinelli R, Georgiou D, Ginzton L, Cianci G, Purcaro A. Effects of moderate exercise training on thallium uptake and contractile response to low-dose dobutamine of dysfunctional myocardium in patients with ischemic cardiomyopathy. Circulation 1998; 97(6): 553-61.

18 - Schuler G, Hambrecht R, Schlierf G, Grunze M, Methfessel S, Hauer K, et al. Myocardial perfusion and regression of coronary artery disease in patients on a regimen of intensive physical exercise and low fat diet. J Am Coll Cardiol 1992; 19(1): 34-42.
19 - Hambrecht R, Wolf A, Gielen S, Linke A, Hofer J, Erbs S, et al. Effect of exercise on coronary endothelial function in patients with coronary artery disease. N Engl J Med 2000; 342(7): 454-60.

20 - Gielen S, Sculer G, Hambrecht R. Exercise training in coronary artery disease and coronary vasomotion. Circulation 2001; 103(1): E1-6.

21 - Van Camp SP, Peterson RA. Cardiovascular complications of outpatient cardiac rehabilitation programs. JAMA 1986; 256(9):1160-3.

22 - Franklin BA, Bonzheim K, Gordon S, Timmis GC. Safety of medically supervised outpatient cardiac rehabilitation exercise therapy. A 16-year follow-up. CHEST 1998; 114 (3): 902-6.

23 - Franklin BA, editor. Diretrizes do American College of Sports Medicine para os testes de esforço e sua prescrição. $6 a$ ed. Rio de Janeiro: Editora Guanabara-Koogan; 2000, p. 1239

24 - Andrade J, coordenador. Diretriz da Sociedade Brasileira de Cardiologia sobre teste ergométrico. Arq Bras Cardiol 2002; 78 (Suppl II): 1-17.

25 - Myers J, Prakash M, Froelicher V, Do D, Partington S, Atwood JE. Exercise capacity and mortality among men referred for exercise testing. N Engl J Med 2002; 346(11): 793-800.

26 - Kavanagh T, Mertens DJ, Hamm LF, Beyene J, Kennedy J, Corey P, et al. Prediction of long term prognosis in 12169 men referred for cardiac rehabilitation. Circulation 2002 106(6): 666-71.

27 - Blair SN, Kampert JB, Kohl HW III, Barlow CE, Macera, CA, Paffenbarger RS Jr, et al. Influences of cardiorespiratory fitness and other precursors on cardiovascular disease and all-cause mortality in men and women. JAMA 1996; 276(3): 205-10.

28 - Steffen-Batey L, Nichaman MZ, Goff DC Jr., Frankowski RF Hanis CL, Ramsey DJ, et al. Change in level of physical activity and risk of all-cause mortality or reinfarction. Circulation 2000; 102(18): 2204-9.

29 - Blair SN, Kohl HW III, Barlow CE, Paffenbarger RS Jr., Gibbons LW, Macera CA. Changes in physical fitness and allcause mortality: a prospective study of healthy and unhealthy men. JAMA 1995; 273(14): 1093-8.

30 - Paffenbarger RS Jr., Hyde RT, Wing AL, Lee I, Jung DL, Kampert JB. The association of changes in physical activity level and other lifestyle characteristics with mortality among men. N Engl J Med 1993; 328(8): 538-45.

Recebido em 15/12/2006

Aprovado em 02/07/2007 